PROCEEDINGS OF THE

AMERICAN MATHEMATICAL SOCIETY

Volume 126, Number 5, May 1998, Pages 1381-1389

S 0002-9939(98)04262-2

\title{
ON THE TOPOLOGICAL BOUNDARY OF SEMI-FREDHOLM OPERATORS
}

\author{
HAÏKEL SKHIRI
}

(Communicated by Palle E. T. Jorgensen)

\begin{abstract}
We prove several distance formulas from a fixed operator in $B(H)$ to some classes of operators connected with the semi-Fredholm ones. Here $H$ is a separable Hilbert space. In particular, Fredholm and upper and lower semi-Fredholm operators have the same boundary in $B(H)$.
\end{abstract}

Let $H$ be a separable Hilbert space and let $B(H)$ be the algebra of all bounded linear oprators on $H$. For an operator $T \in B(H)$, we will denote by $T^{*}, R(T)$, $N(T)$ and $\sigma(T)$ its adjoint, range, kernel and spectrum, respectively. Let $K(H)$ be the ideal of compact operators and $C(H)=B(H) / K(H)$ be the Calkin algebra. Denote by $\pi: B(H) \rightarrow C(H)$ the canonical projection. Endowed with the essential norm $\|T\|_{e}=\|\pi(T)\|, C(H)$ is a $C^{*}$-algebra.

The index of an operator $T \in B(H)$ will be denoted by $\operatorname{ind}(T)$ and is defined by $\operatorname{ind}(T)=\operatorname{dim} N(T)-\operatorname{dim} N\left(T^{*}\right)$, with the convention $\infty-\infty=0$.

We introduce the following notation for several classes of operators :

- $F_{+}=\{T \in B(H): R(T)$ is closed, $\operatorname{dim} N(T)<\infty\}$ is the set of all upper semi-Fredholm operators.

- $F_{-}=\left\{T \in B(H): R(T)\right.$ is closed, $\left.\operatorname{dim} N\left(T^{*}\right)<\infty\right\}$ is the set of all lower semi-Fredholm operators.

- $F_{ \pm}=F_{+} \cup F_{-}$is the set of semi-Fredholm operators.

- $F=F_{+} \cap F_{-}$is the set of Fredholm operators.

- $I_{n}=\{T \in B(H)$ : ind $(T)=n\}$ with $n \in \overline{\mathbb{Z}}=\mathbb{Z} \cup\{-\infty,+\infty\}$.

- $F_{ \pm}^{n}=F_{ \pm} \cap I_{n}$, with $n \in \overline{\mathbb{Z}}$, the connected component of index $n$ in $F_{ \pm}$.

For a set $X$ in $B(H)$, we will denote by $\operatorname{int} X, \bar{X}$ and $\partial X$ the interior, closure and (topological) boundary, respectively.

For a linear operator $T \in B(H)$, we will denote by $\sigma_{e}(T)=\{\lambda \in \mathbb{C}: T-\lambda I \notin F\}$ the essential spectrum of $T$. Let

$$
m_{e}(T)=\inf \left\{\sigma_{e}(|T|)\right\}
$$

(cf. [1]), where $|T|=\left(T^{*} T\right)^{1 / 2}$, and

$$
M_{e}(T)=\max \left\{m_{e}(T) ; m_{e}\left(T^{*}\right)\right\} .
$$

Using Theorem 1.1 of [4] and Theorem 3.1 of [7], we easily obtain the following result:

Received by the editors April 30, 1996 and, in revised form, October 14, 1996.

1991 Mathematics Subject Classification. Primary 47A53; Secondary 47A55.

Key words and phrases. Hilbert space operators, semi-Fredholm operators, distance formulas.

(C)1998 American Mathematical Society 
Theorem 1. Let $T \in B(H)$ and $n \in \overline{\mathbb{Z}}$. Then

$$
\operatorname{dist}\left(T, F_{ \pm}^{n}\right)= \begin{cases}M_{e}(T) & \text { if } T \notin I_{n} \\ 0 & \text { if } n o t .\end{cases}
$$

We recall the following theorem, which gives a characterization of the boundary of connected components of semi-Fredholm operators.

Theorem 2 ([5], Corollary 1.4). The boundary of $\overline{F_{ \pm}^{j}}$ does not depend on $j$, and, if $\Delta=\partial \overline{F_{ \pm}^{j}}$, then $\Delta=\partial F_{ \pm}^{j}$ and $B(H)=\Delta \cup\left(\bigcup_{j \in \overline{\mathbb{Z}}} F_{ \pm}^{j}\right)$.

Remarks. 1) We have $\Delta=\left\{T \in B(H): M_{e}(T)=0\right\}$. Indeed, using [4], Theorem 2.1 and Corollary 2.2, we obtain

$$
\Delta=\partial F_{ \pm}^{0}=\partial \bar{G}=\left\{T \in B(H): M_{e}(T)=0\right\},
$$

where $G$ is the group of invertible operators in $B(H)$.

2) The set $\Delta$ is stable with respect to compact perturbations: $\Delta+K(H)=\Delta$.

3) The set $\Delta$ is arcwise connected. Indeed, if $0 \in \Delta$ and $T \in \Delta$, then $t T \in \Delta$ for all $t \in[0,1]$.

We prove now the following result.

Theorem 3. Let $T \in B(H)$ and $J \subseteq \overline{\mathbb{Z}}$. Then

$$
\text { (a) } \quad \operatorname{dist}\left(T, \bigcup_{j \in J} F_{ \pm}^{j}\right)= \begin{cases}M_{e}(T) & \text { if } \operatorname{ind}(T) \notin J, \\ 0 & \text { if not, }\end{cases}
$$

and

$$
\text { (b) } \quad \operatorname{dist}\left(T, \bigcup_{j \in J} I_{j}\right)= \begin{cases}M_{e}(T) & \text { if } \operatorname{ind}(T) \notin J \\ 0 & \text { if not. }\end{cases}
$$

Proof. (a) Let $n=\operatorname{ind}(T) \in \overline{\mathbb{Z}}$. If $n \in J$, then $F_{ \pm}^{n} \subseteq \bigcup_{j \in J} F_{ \pm}^{j}$. Therefore

$$
0 \leq \operatorname{dist}\left(T, \bigcup_{j \in J} F_{ \pm}^{j}\right) \leq \operatorname{dist}\left(T, F_{ \pm}^{n}\right)
$$

But, using Theorem 1, we obtain $\operatorname{dist}\left(T, F_{ \pm}^{n}\right)=0$. Hence, $\operatorname{dist}\left(T, \bigcup_{j \in J} F_{ \pm}^{j}\right)=0$.

Suppose now that $n \notin J$ and let $j_{0} \in J$. Then, using once again Theorem 1 and the relation $n \neq j_{0}$, we get

$$
\operatorname{dist}\left(T, \bigcup_{j \in J} F_{ \pm}^{j}\right) \leq \operatorname{dist}\left(T, F_{ \pm}^{j_{0}}\right)=M_{e}(T) .
$$


We now show the converse inequality. For every $S \in \bigcup_{j \in J} F_{ \pm}^{j}$, we have $\operatorname{ind}(S) \neq n=$ $\operatorname{ind}(T)$. Then Theorem 1.1 of [6] implies $\|T-S\| \geq M_{e}(T)$. Therefore,

$$
\operatorname{dist}\left(T, \bigcup_{j \in J} F_{ \pm}^{j}\right) \geq M_{e}(T)
$$

and (a) is proved.

In order to prove (b), it suffices to remark that $\bigcup_{j \in J} F_{ \pm}^{j} \subseteq \bigcup_{j \in J} I_{j}$ and to use (a). Indeed, if $\operatorname{ind}(T)=n \in J$, then, using (a), we obtain

$$
0 \leq \operatorname{dist}\left(T, \bigcup_{j \in J} I_{j}\right) \leq \operatorname{dist}\left(T, \bigcup_{j \in J} F_{ \pm}^{j}\right)=0
$$

On the other hand, if $n \notin J$, then for all $S \in \bigcup_{j \in J} I_{j}$, we have $\|T-S\| \geq M_{e}(T)$ (cf. [6], Theorem 1.1). This yields

$$
\operatorname{dist}\left(T, \bigcup_{j \in J} I_{j}\right) \geq M_{e}(T)
$$

Using (a) again, we also find that

$$
\operatorname{dist}\left(T, \bigcup_{j \in J} I_{j}\right) \leq \operatorname{dist}\left(T, \bigcup_{j \in J} F_{ \pm}^{j}\right)=M_{e}(T),
$$

which completes the proof.

As consequences, the results below easily follow. Assertions (b) and (c) of the following corollary are also in [2, Theorems 12 and 13].

Corollary 4. Let $T \in B(H)$. Then:

$$
\begin{aligned}
& \text { (a) } \operatorname{dist}(T, F)= \begin{cases}M_{e}(T) & \text { if } \operatorname{ind}(T)= \pm \infty, \\
0 & \text { if } \text { not. }\end{cases} \\
& \text { (b) } \operatorname{dist}\left(T, F_{+}\right)= \begin{cases}m_{e}\left(T^{*}\right) & \text { if } \operatorname{ind}(T)=+\infty, \\
0 & \text { if } \operatorname{ind}(T) \neq+\infty .\end{cases} \\
& \text { (c) } \operatorname{dist}\left(T, F_{-}\right)= \begin{cases}m_{e}(T) & \text { if } \operatorname{ind}(T)=-\infty, \\
0 & \text { if } \operatorname{ind}(T) \neq-\infty .\end{cases}
\end{aligned}
$$

Proof. (a) follows from Theorem 3 above with $J=\mathbb{Z}$, while (b) and (c) follow from $[2$, Lemma 7] and Theorem 3 above with $J=\mathbb{Z} \cup\{-\infty\}$ and $J=\mathbb{Z} \cup\{+\infty\}$, respectively. 
Now we can prove the following equalities.

Theorem 5. Let $J \subseteq \overline{\mathbb{Z}}$. Then

$$
\begin{gathered}
\text { (a) } \overline{\bigcup_{j \in J} F_{ \pm}^{j}}=\Delta \cup\left(\bigcup_{j \in J} F_{ \pm}^{j}\right)=\bigcup_{j \in J} \overline{F_{ \pm}^{j}} ; \\
\text { (b) } \quad \partial\left(\bigcup_{j \in J} F_{ \pm}^{j}\right)=\Delta .
\end{gathered}
$$

Proof. (a) We prove the first equality. If $J=\overline{\mathbb{Z}}$, then the result follows from Theorem 2 since $\overline{\bigcup_{j \in J} F_{ \pm}^{j}}=B(H)$.

Suppose that $J \neq \overline{\mathbb{Z}}$. Then, using Theorem 2 again, we have

$$
B(H)=\Delta \cup\left(\bigcup_{j \in J} F_{ \pm}^{j}\right) \cup\left(\bigcup_{j \in \overline{\mathbb{Z} \backslash J}} F_{ \pm}^{j}\right) .
$$

It follows that $\Delta \cup\left(\bigcup_{j \in J} F_{ \pm}^{j}\right)$ is closed, being the complement of the open set $\left(\bigcup_{j \in \overline{\mathbb{Z}} \backslash J} F_{ \pm}^{j}\right)$ in $B(H)$. Therefore

$$
\overline{\bigcup_{j \in J} F_{ \pm}^{j}} \subseteq \Delta \cup\left(\bigcup_{j \in J} F_{ \pm}^{j}\right)
$$

In order to show the other inclusion, it suffices to see that $\Delta \subset \overline{\bigcup_{j \in J} F_{ \pm}^{j}}$ (see Theorem $3)$.

The second equality follows from

$$
\bigcup_{j \in J} \overline{F_{ \pm}^{j}}=\bigcup_{j \in J}\left(\Delta \cup F_{ \pm}^{j}\right)
$$

(b) Since $\bigcup_{j \in J} F_{ \pm}^{j}$ is open in $B(H)$, we have

$$
\partial\left(\bigcup_{j \in J} F_{ \pm}^{j}\right)=\left(\overline{\bigcup_{j \in J} F_{ \pm}^{j}}\right) \backslash\left(\bigcup_{j \in J} F_{ \pm}^{j}\right)=\left(\Delta \cup\left(\bigcup_{j \in J} F_{ \pm}^{j}\right)\right) \backslash\left(\bigcup_{j \in J} F_{ \pm}^{j}\right)=\Delta .
$$

The proof is complete.

This easily implies the following consequence:

Corollary 6. We have

(a) $\bar{F}=F \cup \Delta ; \overline{F_{+}}=F_{+} \cup \Delta$ and $\overline{F_{-}}=F_{-} \cup \Delta$;

(b) $\partial F=\partial F_{+}=\partial F_{-}=\Delta$.

We also have

Corollary 7. Let $J \subseteq \overline{\mathbb{Z}}$. Then

$$
\overline{\bigcup_{j \in J} I_{j}}=\Delta \cup\left(\bigcup_{j \in J} F_{ \pm}^{j}\right)=\Delta \cup\left(\bigcup_{j \in J} I_{j}\right)
$$


Proof. Using Theorems 3 and 5, we obtain

$$
\Delta \cup\left(\bigcup_{j \in J} I_{j}\right) \subseteq \overline{\bigcup_{j \in J} I_{j}}=\overline{\bigcup_{j \in J} F_{ \pm}^{j}}=\Delta \cup\left(\bigcup_{j \in J} F_{ \pm}^{j}\right) \subseteq \Delta \cup\left(\bigcup_{j \in J} I_{j}\right)
$$

For the interior and for the boundary of the closure of sets considered in Theorem 5 , we have

Theorem 8. Let $J \subseteq \overline{\mathbb{Z}}, J \neq \overline{\mathbb{Z}}$. Then

$$
\begin{gathered}
\text { (a) } \quad \operatorname{int}\left(\overline{\bigcup_{j \in J} F_{ \pm}^{j}}\right)=\bigcup_{j \in J} F_{ \pm}^{j}=\operatorname{int}\left(\bigcup_{j \in J} \overline{F_{ \pm}^{j}}\right) ; \\
\text { (b) } \partial\left(\overline{\bigcup_{j \in J} F_{ \pm}^{j}}\right)=\partial\left(\bigcup_{j \in J} \overline{F_{ \pm}^{j}}\right)=\Delta .
\end{gathered}
$$

Proof. (a) We show the first equality. The inclusion

$$
\bigcup_{j \in J} F_{ \pm}^{j} \subseteq \operatorname{int}\left(\overline{\bigcup_{j \in J} F_{ \pm}^{j}}\right)
$$

is clear. In order to show the other one, let $T \in \operatorname{int}\left(\overline{\bigcup_{j \in J} F_{ \pm}^{j}}\right)$. If $T$ is semi-Fredholm, then $T \in \bigcup_{j \in J} F_{ \pm}^{j}$. Indeed, using Theorem 5, we have

$$
\begin{aligned}
T \in \operatorname{int}\left(\overline{\bigcup_{j \in J} F_{ \pm}^{j}}\right) & =\operatorname{int}\left(\Delta \cup\left[\bigcup_{j \in J} F_{ \pm}^{j}\right]\right) \\
& \subset \Delta \cup\left(\bigcup_{j \in J} F_{ \pm}^{j}\right)
\end{aligned}
$$

and, because $T \notin \Delta$, we get $T \in \bigcup_{j \in J} F_{ \pm}^{j}$.

Suppose now that $T$ is not semi-Fredholm. Then $T \in \Delta$. Consider $n \notin J$ (this is always possible since $J \neq \overline{\mathbb{Z}}$ ). Using Theorem 2 , we obtain $T \in \Delta=\partial F_{ \pm}^{n}$. Therefore

$$
T \in \operatorname{int}\left(\overline{\bigcup_{j \in J} F_{ \pm}^{j}}\right) \cap \partial F_{ \pm}^{n}
$$

which implies the non-voidness of $\left(\overline{\bigcup_{j \in J} F_{ \pm}^{j}}\right) \cap F_{ \pm}^{n}$. The continuity of the index yields a contradiction.

The second equality follows from Theorem 5 . 
(b) Using (a) and Theorem 5, we obtain

$$
\begin{aligned}
\partial\left(\bigcup_{j \in J} \overline{F_{ \pm}^{j}}\right) & =\partial\left(\overline{\bigcup_{j \in J} F_{ \pm}^{j}}\right) \\
& =\left(\overline{\bigcup_{j \in J} F_{ \pm}^{j}}\right) \backslash \operatorname{int}\left(\overline{\bigcup_{j \in J} F_{ \pm}^{j}}\right) \\
& =\left(\Delta \cup\left[\bigcup_{j \in J} F_{ \pm}^{j}\right]\right) \backslash\left(\bigcup_{j \in J} F_{ \pm}^{j}\right)=\Delta .
\end{aligned}
$$

The proof is complete.

The following consequence can be easily obtained.

Corollary 9. We have

(a) $\operatorname{int}(\bar{F})=F ; \operatorname{int}\left(\overline{F_{+}}\right)=F_{+}$and $\operatorname{int}\left(\overline{F_{-}}\right)=F_{-} ;$

and

(b) $\partial \bar{F}=\partial F=\partial \overline{F_{+}}=\partial F_{+}=\partial \overline{F_{-}}=\partial F_{-}=\Delta$.

We also have

Corollary 10. Let $J \subset \overline{\mathbb{Z}}, J \neq \overline{\mathbb{Z}}$. Then

(a) $\quad \operatorname{int}\left(\overline{\bigcup_{j \in J} I_{j}}\right)=\bigcup_{j \in J} F_{ \pm}^{j}=\operatorname{int}\left(\bigcup_{j \in J} I_{j}\right) ;$

$$
\partial\left(\overline{\bigcup_{j \in J} I_{j}}\right)=\partial\left(\bigcup_{j \in J} I_{j}\right)=\Delta .
$$

Proof. (a) The first equality follows from Corollary 7 and Theorems 5 and 8 .

For the second equality, it is sufficient to note that

$$
\bigcup_{j \in J} F_{ \pm}^{j} \subseteq \operatorname{int}\left(\bigcup_{j \in J} I_{j}\right) \subseteq \operatorname{int}\left(\overline{\bigcup_{j \in J} F_{ \pm}^{j}}\right)=\bigcup_{j \in J} F_{ \pm}^{j} .
$$

Indeed, the first two inclusions are obvious and the last equality is (a).

(b) is a direct consequence of (a) and of Corollary 7 .

We introduce the following notation:

- $G_{+}=\{T \in B(H): T$ is left invertible $\}$.

- $G_{-}=\{T \in B(H): T$ is right invertible $\}$.

- $G_{ \pm}=G_{+} \cup G_{-}$: the set of one-sided invertible operators.

- $G=G_{+} \cap G_{-}$: the set of invertible operators.

For $n \in \overline{\mathbb{Z}}$, let $G_{ \pm}^{n}=G_{ \pm} \cap I_{n}$. For $n \in \overline{\mathbb{Z}}_{-}=\mathbb{Z}_{-} \cup\{-\infty\}$, we denote $G_{+}^{n}=G_{+} \cap I_{n}$, while for $n \in \overline{\mathbb{Z}}_{+}=\mathbb{Z}_{+} \cup\{+\infty\}$, we set $G_{-}^{n}=G_{-} \cap I_{n}$.

Theorem 11. Let $T \in B(H)$ and $J \subseteq \overline{\mathbb{Z}}$. Then

$$
\operatorname{dist}\left(T, \bigcup_{j \in J} G_{ \pm}^{j}\right)= \begin{cases}M_{e}(T) & \text { if } \operatorname{ind}(T) \notin J, \\ 0 & \text { if not. }\end{cases}
$$


Proof. Let $n=\operatorname{ind}(T)$. If $n \in J$, then $G_{ \pm}^{n} \subseteq \bigcup_{j \in J} G_{ \pm}^{j}$, so, using Theorem 3.1 of [7], we have

$$
0 \leq \operatorname{dist}\left(T, \bigcup_{j \in J} G_{ \pm}^{j}\right) \leq \operatorname{dist}\left(T, G_{ \pm}^{n}\right)=0 .
$$

Suppose now that $n \notin J$. Let $j_{0} \in J$. Using Theorem 3.1 of [7], we get

$$
0 \leq \operatorname{dist}\left(T, \bigcup_{j \in J} G_{ \pm}^{j}\right) \leq \operatorname{dist}\left(T, G_{ \pm}^{j_{0}}\right)=M_{e}(T) .
$$

On the other hand, for all $L \in \bigcup_{j \in J} G_{ \pm}^{j}, \operatorname{ind}(L) \neq n=\operatorname{ind}(T)$. Therefore, using Theorem 1.1 of [6], we have $\|T-L\| \geq M_{e}(T)$. Thus

$$
\operatorname{dist}\left(T, \bigcup_{j \in J} G_{ \pm}^{j}\right) \geq M_{e}(T) .
$$

Now (1) and (2) imply the desired equality.

We obtain the following consequence.

Corollary 12. Let $T \in B(H)$, and $J \subseteq \overline{\mathbb{Z}}_{+}$. Then

$$
\operatorname{dist}\left(T, \bigcup_{j \in J} G_{-}^{j}\right)= \begin{cases}M_{e}(T) & \text { if } \operatorname{ind}(T) \notin J, \\ 0 & \text { if } \operatorname{ind}(T) \in J .\end{cases}
$$

A similar result can be stated for $\operatorname{dist}\left(T, \bigcup_{j \in J} G_{+}^{j}\right)$ if $J \subseteq \overline{\mathbb{Z}}_{-}$. For the distance of $T$ to the set $G_{-} \backslash G$ we obtain the following formula:

Corollary 13. Let $T \in B(H)$. Then

$$
\operatorname{dist}\left(T, G_{-} \backslash G\right)= \begin{cases}M_{e}(T) & \text { if } \operatorname{ind}(T) \leq 0, \\ 0 & \text { if not. }\end{cases}
$$

The following result gives a description of the closure, the interior and the boundary of $\bigcup_{j \in J} G_{ \pm}^{j}$ for $J \subseteq \overline{\mathbb{Z}}$.

Theorem 14. Let $J \subseteq \overline{\mathbb{Z}}$. Then:

(a) $\overline{\bigcup_{j \in J} G_{ \pm}^{j}}=\Delta \cup\left(\bigcup_{j \in J} F_{ \pm}^{j}\right)=\bigcup_{j \in J} \overline{G_{ \pm}^{j}}$.

If in addition $J \neq \overline{\mathbb{Z}}$, then:

(b) $\operatorname{int}\left(\overline{\bigcup_{j \in J} G_{ \pm}^{j}}\right)=\bigcup_{j \in J} F_{ \pm}^{j}=\operatorname{int}\left(\bigcup_{j \in J} \overline{G_{ \pm}^{j}}\right)$,

(c) $\partial\left(\overline{\bigcup_{j \in J} G_{ \pm}^{j}}\right)=\partial\left(\bigcup_{j \in J} \overline{G_{ \pm}^{j}}\right)=\Delta$. 
Proof. (a) We prove the first equality. If $J=\overline{\mathbb{Z}}$, then, using [3, Problem 109], $G_{ \pm}$is dense in $B(H)$ and the result follows from Theorem 2 .

Suppose now that $J \neq \overline{\mathbb{Z}}$. Using Theorem 5 , we obtain that $\Delta \cup\left(\bigcup_{j \in J} F_{ \pm}^{j}\right)$ is closed. But $\bigcup_{j \in J} G_{ \pm}^{j} \subseteq \Delta \cup\left(\bigcup_{j \in J} F_{ \pm}^{j}\right)$. Thus

$$
\overline{\bigcup_{j \in J} G_{ \pm}^{j}} \subseteq \Delta \cup\left(\bigcup_{j \in J} F_{ \pm}^{j}\right) .
$$

On the other hand, using Theorem 11, we have:

$$
\Delta \cup\left(\bigcup_{j \in J} F_{ \pm}^{j}\right) \subseteq \overline{\bigcup_{j \in J} G_{ \pm}^{j}}
$$

The first equality follows from (1) and (2).

The second equality follows from $\overline{G_{ \pm}^{j}}=\overline{F_{ \pm}^{j}}$, for all $j \in \overline{\mathbb{Z}}$. This follows easily from Theorem 1 and Theorem 11.

(b) is a direct consequence of (a), Theorem 5 and Theorem 8.

(c) Since the first equality follows from (a) and (b), it sufficient to show the second one. Using (a) and (b), we have

$$
\partial\left(\bigcup_{j \in J} \overline{G_{ \pm}^{j}}\right)=\left(\Delta \cup\left[\bigcup_{j \in J} F_{ \pm}^{j}\right]\right) \backslash\left(\bigcup_{j \in J} F_{ \pm}^{j}\right)=\Delta .
$$

The proof is complete.

We obtain as consequences the following formulas:

Corollary 15.1) a) $\overline{G_{+}}=\Delta \cup\left(\bigcup_{j \leq 0} F_{ \pm}^{j}\right)$,

b) $\overline{G_{-}}=\Delta \cup\left(\bigcup_{j \geq 0} F_{ \pm}^{j}\right)$;

2) a) $\operatorname{int}\left(\overline{G_{+}}\right)=\bigcup_{j \leq 0}^{j \geq 0} F_{ \pm}^{j}$,

b) $\operatorname{int}\left(\overline{G_{-}}\right)=\bigcup_{j \geq 0}^{j \leq 0} F_{ \pm}^{j}$;

3) $\partial \overline{G_{+}}=\partial \overline{G_{-}}=\Delta$.

Corollary 16. 1) a) $\overline{G_{+} \backslash G}=\Delta \cup\left(\bigcup_{j<0} F_{ \pm}^{j}\right)$,

b) $\overline{G_{-} \backslash G}=\Delta \cup\left(\bigcup_{j>0} F_{ \pm}^{j}\right)$;

2) a) $\operatorname{int}\left(\overline{G_{+} \backslash G}\right)=\bigcup_{j<0} F_{ \pm}^{j}$,

b) $\operatorname{int}\left(\overline{G_{-} \backslash G}\right)=\bigcup_{j>0}^{j<0} F_{ \pm}^{j}$;

3) $\partial\left(\overline{G_{+} \backslash G}\right)=\partial\left(\overline{G_{-} \backslash G}\right)=\Delta$.

Similar formulas can be given for $\bigcup_{j \in J} G_{+}^{j}, J \subseteq \overline{\mathbb{Z}}_{-}$, and for $\bigcup_{j \in J} G_{-}^{j}, J \subseteq \overline{\mathbb{Z}}_{+}$. 


\section{ACKNOWLEDGEMENT}

I would like to thank my supervisor, M. Mbekhta, for his guidance, and C. Badea for his help.

\section{REFERENCES}

[1] R. Bouldin, The essential minimum modulus, Indiana. Univ. Math. J. 30 (1981), 513-517. MR 82i: 47001

[2] R. Bouldin, Approximation by semi-Fredholm operators with fixed nullity, Rocky Mountain J. Math. 20 (1990), 39-50. MR 91m:47021

[3] P.R. Halmos, A Hilbert space problem book, D. Van Nostrand, 1967. MR 34:8178

[4] S. Izumino \& Y. Kato, The closure of invertible operators on a Hilbert space, Acta. Sci. Math. (Szeged) 49 (1985), 321-327. MR 87h:47101

[5] M. Mbekhta, Sur la structure des composantes connexes semi-Fredholm de B(H), Proc. Amer. Math. Soc. 116 (1992), 521-524. MR 92m:48021

[6] D.D. Rogers, Approximation by unitary and essentially unitary operators, Acta. Sci. Math. (Szeged) 39 (1977), 141-151. MR 56:6439

[7] P. Y. Wu, Approximation by invertible and noninvertible operators, J. Approx. Theory, 56 (1989), 267-276. MR 90c:47003

Département de Mathématiques, Bât. M2, Université de Lille I, F-59655 Villeneuve D'ASCQ, France

E-mail address: skhiri@gat.univ-lille1.fr 